\title{
Comparison of Patient-Led and Physician-Led Insulin Titration in Japanese Type 2 Diabetes Mellitus Patients Based on Treatment Distress, Satisfaction, and Self- Efficacy: The COMMIT-Patient Study
}

\author{
Hitoshi Ishii (D) · Hiroki Nakajima · Nozomu Kamei · Daigaku Uchida • \\ Daisuke Suzuki · Yasuhiro Ono · Yasunori Sato $\cdot$ Dai Shimono
}

Received: December 1, 2020 / Accepted: December 23, 2020 / Published online: January 18, 2021

(C) The Author(s) 2021

\begin{abstract}
Introduction: In Japan, patient-led insulin titration is rare in type 2 diabetes mellitus (T2DM) patients. Few studies have compared the effects of patient-led versus physician-led insulin titration on patient-reported outcomes in Japanese T2DM patients. This study aimed to compare the effects of patient-led and physician-led insulin titration in Japanese insulinnaïve T2DM patients on safety, glycemic control, and patient-reported outcomes (emotional distress, treatment satisfaction, and selfefficacy).
\end{abstract}

Supplementary Information The online version contains supplementary material available at https:// doi.org/10.1007/s13300-020-00995-8.

H. Ishii $(\bowtie)$

Department of Doctor-Patient Relationships, Nara

Medical University, Kashihara, Nara, Japan

e-mail: hit3910@gmail.com

\section{H. Nakajima}

Department of Diabetes and Endocrine Medicine,

Nara Medical University, Kashihara, Nara, Japan

N. Kamei

Department of Endocrinology and Metabolism, Hiroshima Red Cross Hospital \& Atomic Bomb Survivors Hospital, Hiroshima, Hiroshima, Japan

D. Uchida

Hotaruno Central Naika, Kisarazu, Chiba, Japan
Methods: Ultimately, 125 insulin-naïve Japanese T2DM patients were randomly assigned to either a patient-led insulin self-titration group or a physician-led insulin titration group and monitored for 24 weeks. The primary endpoint was a change in emotional distress as measured using the Problem Areas in Diabetes scale (PAID). Secondary endpoints included treatment satisfaction, as measured with the Diabetes Treatment Satisfaction Questionnaire (DTSQ), self-efficacy as measured using the Insulin Therapy Self-Efficacy Scale (ITSS), glycated hemoglobin (HbA1c) levels, fasting plasma glucose levels, body weight, insulin daily dose, and frequency of hypoglycemia.

Results: There was no significant difference between the groups in PAID and DTSQ scores. The results for the primary endpoint should be interpreted taking account that the sample size

\author{
D. Suzuki \\ STOP DM Suzuki Diabetes Clinic, Atsugi, Kanagawa, \\ Japan \\ Y. Ono \\ Takagi Hospital, Okawa, Fukuoka, Japan \\ Y. Sato \\ Department of Preventive Medicine and Public \\ Health, Keio University School of Medicine, Tokyo, \\ Japan \\ D. Shimono \\ Futata Tetsuhiro Clinic, Fukuoka, Fukuoka, Japan
}


for the power calculation was not reached. ITSS scores were significantly higher in the patientled self-titration group. HbA1c and fasting plasma glucose levels were significantly decreased in both groups, but the decrease was significantly larger in the patient-led self-titration group. Although the insulin daily dose was significantly higher in the patient-led self-titration group, severe hypoglycemia did not occur in either group, and the frequency of hypoglycemia was similar in both groups.

Conclusion: Self-measurement of blood glucose and self-titration of insulin enhanced the patients' self-efficacy without compromising their emotional distress or treatment satisfaction. Also, insulin self-titration was found to be safe and effective; it resulted in better glycemic control without severe hypoglycemia.

Trial registration: University Hospital Medical Information Network Clinical Trials Registry (UMIN-CTR) (registration number: UMIN000020316).

Keywords: Emotional distress; Insulin; Patientled insulin self-titration; Patient-reported outcome; Physician-led insulin titration; Quality of life; Self-efficacy; Self-management; Treatment satisfaction

\section{Key Summary Points}

\section{Why carry out this study?}

Few studies have compared the patientreported outcomes, including emotional distress, treatment satisfaction, and selfefficacy, of physician-led insulin titration with those of patient-led insulin selftitration.

This study enrolled insulin-naïve patients with type 2 diabetes mellitus and compared physician-led insulin titration to patient-led insulin self-titration in terms of emotional distress, treatment satisfaction, and self-efficacy.

\section{What was learned from the study?}

Self-efficacy was significantly higher in the patient-led self-titration group.
Self-measurement of blood glucose and self-titration of insulin was safe and effective in Japanese patients with type 2 diabetes mellitus, resulting in better glycemic control without severe hypoglycemia.

Self-management of diabetes, including self-measurement of blood glucose and insulin self-titration, may help combat clinical inertia regarding insulin treatment in patients with type 2 diabetes mellitus.

\section{DIGITAL FEATURES}

This article is published with digital features, including a summary slide, to facilitate understanding of the article. To view digital features for this article go to https://doi.org/10.6084/ m9.figshare.13476858.

\section{INTRODUCTION}

Insulin treatment is an important and effective means of glycemic control for both type 1 (T1DM) and type 2 (T2DM) diabetes mellitus (DM) patients. Insulin titration based on blood glucose levels is crucial to achieving favorable glycemic control. Insulin titration is primarily conducted in one of two ways: physician-led titration or patient-led self-titration. Patient-led self-titration is commonly used in T1DM, but less so in T2DM, especially in Asian countries such as Japan [1, 2]. However, several clinical trials have demonstrated that the results of patient-led self-titration based on self-measurement of blood glucose (SMBG) are comparable to glycemic control with physician-led titration [2-5]. These results suggest that patient-led insulin self-management, including self-titration, is effective at managing T2DM.

Self-management is an important factor when managing DM. SMBG and insulin self-titration may empower patients, resulting in greater engagement with their therapy and enhancing their self-confidence/self-efficacy [3]. 
The confidence that self-management can instill in a patient's treatment may improve their quality-of-life (QOL) and treatment satisfaction. However, few studies have measured such patient-reported outcomes (PROs), including QOL, treatment satisfaction, and selfefficacy. One such study, the ATLAS study [2], found no difference between patient-led and physician-led T2DM patients in treatment satisfaction and QOL as measured using the Diabetes Treatment Satisfaction Questionnaire (DTSQ) [6] and the EuroQol instrument (EQ-5D) [7], respectively.

To address the lack of clinical trials reporting PROs, the Comparison of Satisfaction Between Patient-Led and Physician-Led Titration Groups Measured by QOL and Self-Efficacy Scores In Japanese T2D Patients (COMMIT-patient) study was conducted to compare patient-led and physician-led insulin titration in terms of safety, clinical outcomes, and PROs. PROs were measured using the Problem Areas in Diabetes (PAID) scale $[8,9]$, the DTSQ $[6,10]$, and the Insulin Therapy Self-Efficacy Scale (ITSS) [11]. Since insulin treatment is associated with greater distress than other antidiabetic therapies $[9,12,13]$, we hypothesized that insulin selftitration, which improves the self-recognition of treatment goals and requires greater self-involvement in therapy, may reduce insulin injection-related distress in patients. Our previous study demonstrated that treatment satisfaction was lower in patients receiving insulin than in those receiving other antidiabetic treatments. The ATLAS study [2] of an Asian population reported no difference in treatment satisfaction between physician-led and patientled titration, while glycemic control was significantly more improved in the patient-led group. We sought to investigate whether patient-led insulin titration was available for Japanese patients, and the effects of patient-led and physician-led insulin titration on PROs. Since the ultimate goal of insulin self-titration is to develop the patient's self-efficacy regarding self-management of their DM, we compared ITSS scores between physician-led and patientled titration in T2DM patients in this study. By determining the optimal management strategy for T2DM, we can reduce the psychological burden of insulin therapy, improve treatment satisfaction, and improve patient confidence in insulin therapy.

\section{METHODS}

\section{Study Design}

The COMMIT-patient study was a prospective, randomized, open-label, blinded-endpoint trial conducted across 30 medical institutions in Japan between January 2016 and January 2020 . This study is registered in the University Hospital Medical Information Network Clinical Trials Registry (UMIN-CTR) (registration number: UMIN000020316), and was conducted in accordance with the principles of the Declaration of Helsinki, the Ethical Guidelines for Medical and Health Research Involving Human Subjects in Japan, and other relevant bylaws and regulations. The study protocol was first approved by the Nara Medical University Ethics Committee, and then by the ethics committees for all the other institutions involved in this study. The names of all participating research institutions and those of the ethics committees that approved the study are listed in Table S1 of the "Supplementary Information". Written informed consent was obtained prior to treatment from all enrolled patients who met the eligibility criteria. All authors had access to the study data and reviewed and approved the final manuscript.

\section{Patient Population}

T2DM patients who were initiating insulin therapy were included in this study. The main inclusion criteria were as follows: (1) newly starting insulin therapy and able to self-administer (self-inject) insulin, (2) men and women aged 20 years or older, (3) diagnosis of T2DM for at least 6 months, (4) previous use of oral hypoglycemic agents (OHAs) for at least 3 months or longer, and (5) HbA1c levels of between 7 and 10\%. The main exclusion criteria were as follows: (1) dementia (diagnosed or suspected) or other psychological conditions, 
(2) insufficient cognitive and judging ability or an inability to read and write, (3) inability to personally give informed consent, and (4) visual impairment that interfered with self-injection. Further eligibility criteria are summarized in Table S2 of the "Supplementary Information".

\section{Randomization and Study Intervention}

After obtaining informed consent, eligible patients were randomly assigned to either the patient-led group or the physician-led group at a ratio of approximately 1:1. Randomization was performed using a computer-based dynamic allocation method with a minimization procedure to balance three allocation factors (age, gender, and daily number of hypoglycemic agent administrations) across the groups. All enrolled subjects started insulin glargine U300 at an initial dose of $0.1 \times$ body weight $(\mathrm{kg})$. The insulin titration algorithm for each group is summarized in Table S3 of the "Supplementary Information". Antidiabetic agents or other therapeutic agents used at giving their consent were allowed to continue to use during the study, but changes in antidiabetic agent dosage and type were prohibited during the study (except for the study agent, insulin glargine U300). If the patient was using a sulfonylurea when they gave their consent to participate in the study, glimepiride and gliclazide doses were decreased to $1 \mathrm{mg}$ and $40 \mathrm{mg}$, respectively, and glibenclamide was changed to $1 \mathrm{mg}$ of glimepiride for safety reasons at the start of the study. However, changes in sulfonylurea dosage and type were prohibited during the study. Patients were followed up for 24 weeks, with observations performed at baseline and weeks $1,2,4,6,8,12,16,20$, and 24 .

\section{Study Outcomes}

The primary endpoint was the change in PAID score from baseline to week 24 . The secondary endpoints were DTSQ score, ITSS score, HbA1c level, fasting plasma glucose (FPG), body weight, insulin daily dose, frequency of hypoglycemia, frequency of other adverse events, and treatment adherence. The PAID is a questionnaire measuring DM-related distress $[8,9]$, the DTSQ is a questionnaire to measure treatment satisfaction in diabetes care $[6,10]$, and the ITSS is a questionnaire to assess a patient's confidence with their insulin treatment, which consists of four domains: domain 1 , confidence with the insulin injection procedure; domain 2, confidence with the insulin titration; domain 3, confidence with glycemic control; and domain 4, confidence with the ability to deal with hypoglycemia [11].

\section{Data Collection}

Questionnaires about PROs were conducted on paper or on an iPad. Results from clinical laboratory tests were recorded on a paper-based case report form. The data obtained in this study were managed by a third-party entity (Soiken Inc.) to avoid bias, and the statistical analysis was performed by an independent biostatistician (Yasunori Sato, Keio University).

\section{Sample Size Calculation and Statistical Analysis}

Since no previous randomized controlled trial had reported our primary endpoint (i.e., change in PAID score from pre- to- postinitiation of insulin therapy in Japanese T2DM patients), we utilized our unpublished cross-sectional results from using the Japanese version of the PAID scale in Japanese T2DM patients. We observed a mean PAID score of 51.0 in patients who received OHAs, while patients who responded neutrally to the item "Not having clear and concrete goals for your diabetes care?" had a mean PAID score of 55.8. Therefore, we hypothesized that the mean PAID score at baseline would be 51.0, while the mean scores at week 24 in the patient-led group and the physician-led group would be 51.0 (no change from baseline) and 55.0 (an increase of 4 points), respectively, given that the PAID score increases upon insulin initiation and decreases upon achieving the treatment goals. Assuming a standard deviation of 10, 100 patients per group would provide a power of over $80 \%$ to detect a difference in the mean PAID score using 
a two-sided $t$ test at 5\% significance. A dropout rate of approximately $20 \%$ was estimated. Thus, 125 patients were required per group, giving a total required sample size of 250 .

Analyses for the primary and secondary endpoints were primarily performed on the full analysis set (FAS), which included all subjects assigned to a study intervention. The safety analysis included all treated patients. All tests were two-sided and a $p$ value of $<0.05$ was considered statistically significant. The primary and secondary endpoints were assessed by mixed-effects models for repeated measures analysis (MMRM) or analysis of covariance (ANCOVA), with treatment group, time, interaction between treatment groups, allocation factors, and baseline values as fixed effects and subjects nested by treatment group as a random effect. For frequency of hypoglycemia and adverse events, summary statistics were calculated and the chi-squared test or Fisher's exact test was used for between-group comparisons. The SAS statistical software package (version 9.4; SAS, Cary, NC, USA) was used to perform all statistical analyses. All statistical analyses were performed by an independent biostatistician.

\section{RESULTS}

\section{Baseline Characteristics of Study Participants}

In total, 373 patients were screened and 248 were excluded. Of the excluded patients, $41 \mathrm{did}$ not provide consent, 201 did not match the eligibility/exclusion criteria, and 6 were excluded for other reasons. Therefore, 125 patients were enrolled in the study and randomly allocated to a treatment group. One hundred twenty patients completed the study and were included in the FAS, including 59 patients in the patient-led group and 61 subjects in the physician-led group (Fig. 1). The baseline characteristics of the patients are summarized in Table 1. No significant between-group differences in baseline characteristics were found.

\section{Patient-Reported Outcomes}

\section{PAID}

The mean PAID score significantly decreased from baseline to week 12 (changes from baseline were -5.7 (95\% confidence interval (CI) -8.7 to -2.6$)$ and $-4.2(95 \%$ CI -7.1 to - 1.3) in the patient-led and physician-led groups, respectively) and week 24 (changes from baseline were $-6.2(95 \%$ CI -9.4 to - 3.0) and -6.1 (95\% CI - 9.1 to -3.0$)$ in the patient-led and physician-led groups, respectively) in both groups. No significant betweengroup differences were observed for either the baseline to week 12 change or the baseline to week 24 change (between-group differences in change were $-1.4(95 \% \mathrm{CI}-5.3$ to 2.5$)$ $(p=0.472)$ and -0.1 (95\% CI -4.3 to 4.0$)$ $(p=0.947)$ at weeks 12 and 24 , respectively) (Fig. 2).

\section{DTSQ}

The DTSQ score significantly increased from baseline to week 12 (changes from baseline were 3.6 (95\% CI 2.0-5.2) and 1.7 (95\% CI 0.2-3.2) in the patient-led group and the physician-led group, respectively) and week 24 (changes from baseline were 3.2 (95\% CI 1.5-5.0) and 2.1 (95\% CI 0.5-3.7) in the patient-led and physician-led groups, respectively) in both groups. Although the patient-led group showed a tendency towards a greater increase in mean DTSQ score from baseline to week 12 than the physician-led group, no significant between-group differences were observed for either the baseline to week 12 change or the baseline to week 24 change (between-group differences in change were 1.9 $(95 \% \mathrm{CI}-0.2$ to 4.0$)(p=0.074)$ and $1.1(95 \%$ CI -1.1 to 3.4$)(p=0.327)$ at weeks 12 and 24 , respectively) (Fig. 2).

\section{ITSS}

As the ITSS assesses self-efficacy regarding insulin treatment and insulin-naïve patients were enrolled in this study, the ITSS was collected at weeks 12 and 24 but not at baseline. At weeks 12 and 24, the mean ITSS total score was significantly higher in the patient-led group than in the physician-led group $[77.2 \pm 11.6$ 


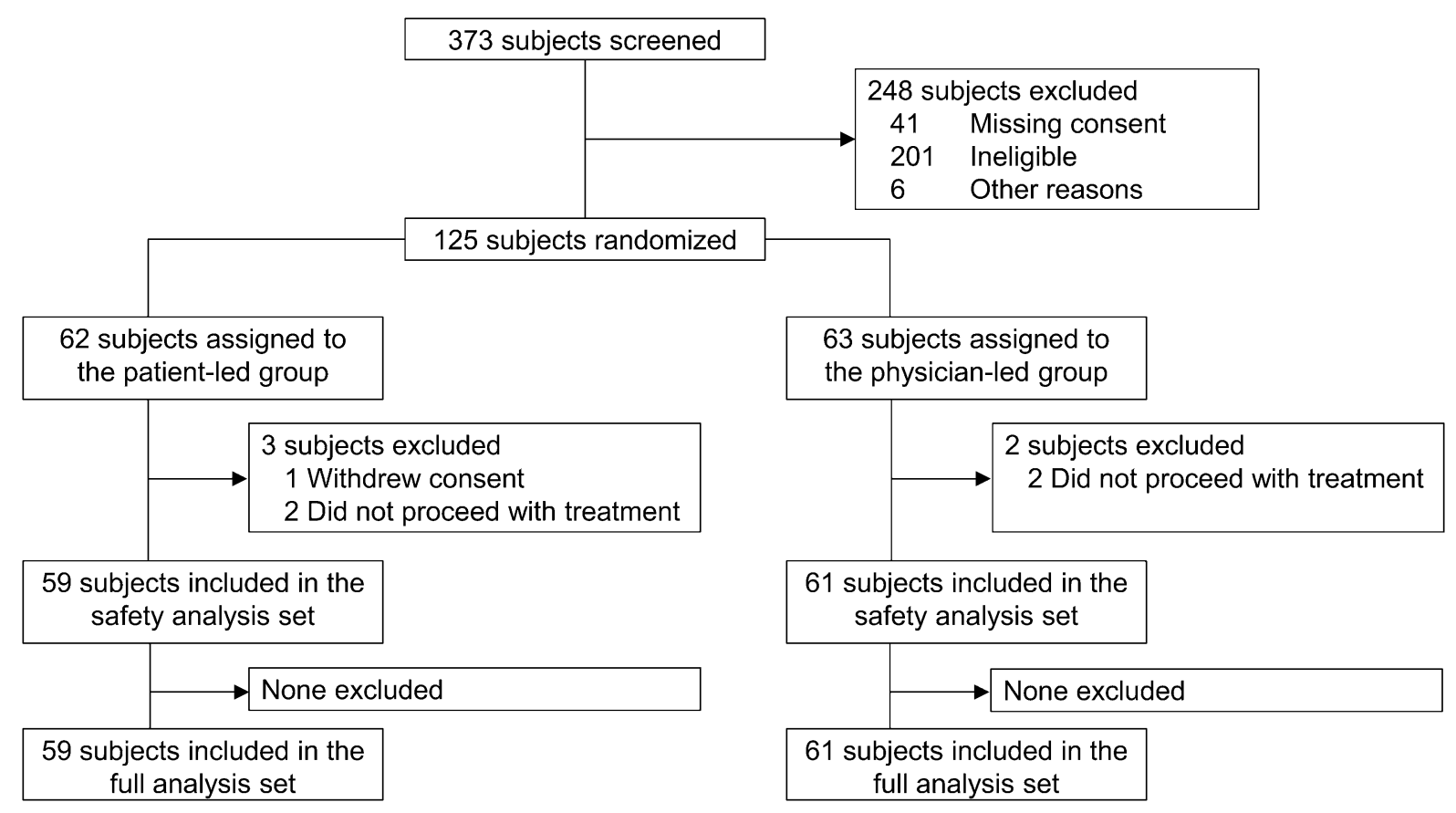

Fig. 1 Study flow chart showing patient enrollment, allocation, and analysis

and $71.1 \pm 12.9$ in the patient-led group and the physician-led group, respectively, at week $12(p=0.009)$ and $77.8 \pm 12.7$ and $71.4 \pm 11.0$ in the patient-led group and the physician-led group, respectively, at week $24(p=0.005)$ ] (Fig. 2). No significant change in the mean ITSS total score was observed between weeks 12 and 24 [changes from week 12 were 0.4 (95\% CI -1.7 to 2.6$)$ and -1.2 (95\% CI -3.3 to 0.8$)$; the between-group difference was 1.7 (95\% CI -1.1 to 4.4$) \quad(p=0.230)]$. Among these domains, the mean domain 2 and domain 3 scores were significantly higher in the patientled group at weeks 12 and 24 [domain 2: $75.4 \pm 17.3$ and $56.5 \pm 26.4$ in the patient-led group and the physician-led group, respectively, at week $12(p<0.001)$ and $76.7 \pm 17.3$ and $56.9 \pm 23.0$ in the patient-led group and the physician-led group, respectively, at week 24 $(p<0.001) ; \quad$ domain $3: \quad 55.9 \pm 18.0$ and $47.2 \pm 17.2$ in the patient-led group and the physician-led group, respectively, at week 12 $(p=0.010)$ and $58.8 \pm 17.2$ and $52.1 \pm 17.9$ in the patient-led group and the physician-led group, respectively, at week $24(p=0.041)$ ] (see Table S4 of the "Supplementary Information").
Specifically, domain 2 scores increased in the patient-led group, whereas the scores decreased in the physician-led group, resulting in the observed significant between-group difference (the between-group difference in change was 7.9 (95\% CI 2.0-13.7), $p=0.009$ ).

\section{Clinical Outcomes}

\section{Glycemic Control}

HbA1c levels decreased significantly from baseline to week 12 (changes from baseline were $-1.4 \%$ (95\% CI -1.6 to $-1.2 \%)$ and $-1.1 \%$ (95\% CI -1.3 to $-0.9 \%)$ in the patient-led group and the physician-led group, respectively) and from baseline to week 24 (changes from baseline were $-1.7 \%(95 \%$ CI -1.9 to $-1.4 \%)$ and $-1.3 \%$ (95\% CI -1.5 to $-1.1 \%)$ in the patient-led group and the physician-led group, respectively) in both groups (Fig. 3). The decrease was significantly larger in the patientled group at week 12 (between-group difference in change was $-0.3 \% \quad(95 \% \mathrm{CI}-0.6$ to $-0.04 \%$ ), $p=0.022$ ) and week 24 (betweengroup difference in change was $-0.4 \%(95 \% \mathrm{CI}$ -0.7 to $-0.1 \%), p=0.014)$. The mean HbA1c 
Table 1 Baseline patient characteristics

\begin{tabular}{|c|c|c|c|}
\hline Characteristic & Patient-led group $(n=59)$ & Physician-led group $(n=61)$ & $p$ value \\
\hline Age (years) & $63.9 \pm 11.6$ & $61.4 \pm 11.5$ & 0.240 \\
\hline Sex (male/female) & $42(71.2) / 17(28.8)$ & $43(70.5) / 18(29.5)$ & 1.000 \\
\hline Height $(\mathrm{cm})$ & $164.6 \pm 9.3$ & $164.3 \pm 10.8$ & 0.859 \\
\hline Body weight $(\mathrm{kg})$ & $64.9 \pm 14.5$ & $66.5 \pm 12.6$ & 0.518 \\
\hline Duration of diabetes (years) & $12.6 \pm 7.4$ & $14.3 \pm 8.6$ & 0.273 \\
\hline Current smoker & $16(27.1)$ & $11(18.0)$ & 0.278 \\
\hline Drinking habit & $28(47.5)$ & $31(50.8)$ & 0.719 \\
\hline Microvascular complications & $35(59.3)$ & $32(52.5)$ & 0.468 \\
\hline Macrovascular complications & $6(10.2)$ & $4(6.6)$ & 0.526 \\
\hline Antidiabetic drugs & $59(100)$ & $61(100)$ & \\
\hline Insulin & $0(0.0)$ & $0(0.0)$ & \\
\hline Other antidiabetic drugs & $59(100)$ & $61(100)$ & \\
\hline Sulfonylurea & $26(44.1)$ & $28(45.9)$ & 0.84 \\
\hline Biguanide & $31(52.5)$ & $36(59.0)$ & 0.48 \\
\hline$\alpha-G I$ & $18(30.5)$ & $17(27.9)$ & 0.75 \\
\hline Glinide & $12(20.3)$ & $14(23.0)$ & 0.73 \\
\hline Thiazolidine & $11(18.6)$ & $8(13.1)$ & 0.41 \\
\hline DPP-4 inhibitor & $52(88.1)$ & $51(83.6)$ & 0.48 \\
\hline SGLT2 inhibitor & $17(28.8)$ & $20(32.8)$ & 0.64 \\
\hline Antihypertensive drugs & $28(47.5)$ & $32(52.5)$ & 0.715 \\
\hline Lipid-lowering drugs & $33(55.9)$ & $43(70.5)$ & 0.130 \\
\hline Antithrombotic drugs & $10(16.9)$ & $13(21.3)$ & 0.645 \\
\hline Other concomitant drugs & $29(49.2)$ & $37(60.7)$ & 0.271 \\
\hline
\end{tabular}

Data are presented as the mean \pm standard deviation or frequency (percentage), as appropriate. $p$ values for between-group comparisons were obtained using Student's $t$ test and Fisher's exact test for continuous and categorical variables, respectively $\alpha-G I$ alpha-glucosidase inhibitor, DPP-4 inhibitor dipeptidyl peptidase-4 inhibitor, SGLT2 inhibitor sodium-glucose cotransporter 2 inhibitor

at week 24 was $6.8 \pm 0.6 \%$ in the patient-led group and $7.2 \pm 1.0 \%$ in the physician-led group, respectively. The proportion of subjects who achieved HbA1c $<7 \%$ was $65.5 \%$ and $47.5 \%$ in the patient-led group and the physician-led group, respectively, indicating that it tended to be higher in the patient-led self-titration group $(p=0.061)$.
Similarly, FPG decreased to $65.1 \mathrm{mg} / \mathrm{dL}$ (95\% CI -75.1 to $-55.0 \mathrm{mg} / \mathrm{dL}$ ) in the patient-led group and $43.3 \mathrm{mg} / \mathrm{dL}(95 \%$ CI -52.7 to $-33.9 \mathrm{mg} / \mathrm{dL}$ ) in the physician-led group from baseline to week 24 . The mean FPG at week 24 was $113.7 \pm 27.8 \mathrm{mg} / \mathrm{dL}(6.3 \mathrm{mmol} / \mathrm{L})$ in the patient-led group and $131.6 \pm 38.8 \mathrm{mg} / \mathrm{dL}$ $(7.3 \mathrm{mmol} / \mathrm{L})$ in the physician-led group 

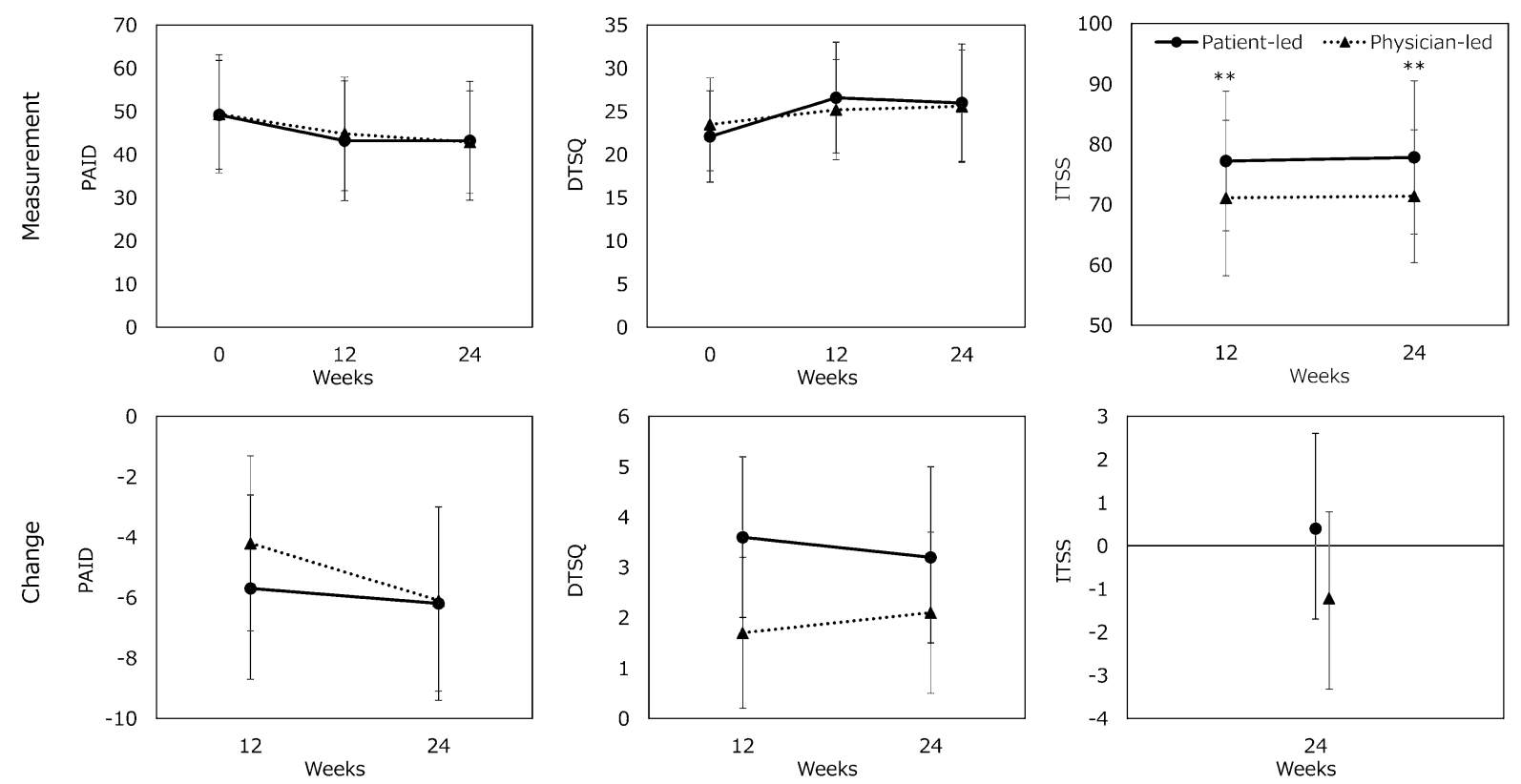

Fig. 2 Measurements and changes in patient-reported outcomes. Data are presented as the mean \pm standard deviation for measurements or the mean $\pm 95 \%$ confidence interval for changes. Changes represent the change from baseline in PAID and DTSQ and the change from week 12 in ITSS. $p$ values for between-group comparisons were obtained using Student's $t$ test for measurements and MMRM for changes in PAID and DTSQ. $p$ values for

(Fig. 3). Especially in the patient-led group, the mean FPG at week 24 almost reached the target glycemic control. The decrease in FPG was significantly larger in the patient-led group at week 12 (between-group difference was $-20.1 \mathrm{mg} / \mathrm{dL}(95 \% \mathrm{CI}-31.0$ to $-9.2 \mathrm{mg} / \mathrm{dL})$, $p<0.001$ ) and week 24 (between-group difference was $-21.8 \mathrm{mg} / \mathrm{dL}(95 \%$ CI -34.9 to $-8.6 \mathrm{mg} / \mathrm{dL}), p=0.001)$.

\section{Insulin Daily Dose and Adherence}

The insulin daily dose was significantly higher in the patient-led group at week 12 $(20.0 \pm 9.7$ unit/day and $14.1 \pm 7.4$ unit/day in the patient-led group and the physician-led group, respectively, $p<0.001$ ) and week 24 $(24.3 \pm 15.6 \mathrm{unit} /$ day and $15.6 \pm 8.5 \mathrm{unit} /$ day in the patient-led group and the physician-led group, respectively, $p<0.001$ ) (Fig. 3). This increase in insulin daily dose was significantly higher in the patient-led group than in the between-group comparisons were obtained by Student's $t$ test for measurements and ANCOVA for the change in ITSS. ${ }^{*},{ }^{* *}$, and ${ }^{* * *}$ represent $p<0.05, p<0.01$, and $p<0.001$, respectively. PAID Problem Areas in Diabetes scale, DTSQ Diabetes Treatment Satisfaction Questionnaire, ITSS Insulin Therapy Self-Efficacy Scale, MMRM mixed-effects models for repeated-measures analysis, $A N C O V A$ analysis of covariance

physician-led group at week 12 (between-group difference was 6.3 unit/day $(95 \%$ CI 3.5-9.0 unit/day), $p<0.001$ ) and week 24 (betweengroup difference was 9.6 unit/day (95\% CI 5.4-13.7 unit/day), $p<0.001$ ).

The insulin self-injection adherence was as high as $>97 \%$ throughout the study in both groups $(97.8 \pm 8.6$ and $97.4 \pm 9.0$ from week 0 to week $12,98.1 \pm 3.8$ and $98.3 \pm 4.8$ from week 12 to week 24 , and $97.4 \pm 8.5$ and $97.6 \pm 7.5$ from week 0 to week 24 in the patient-led and physician-led insulin titration groups, respectively), and no significant between-group difference was observed $(p=0.827,0.851$, and 0.888 for weeks $0-12$, weeks 12-24, and weeks 0-24, respectively) (Table 2).

\section{Body Weight}

In contrast, body weight significantly increased in both groups from baseline to week 12 

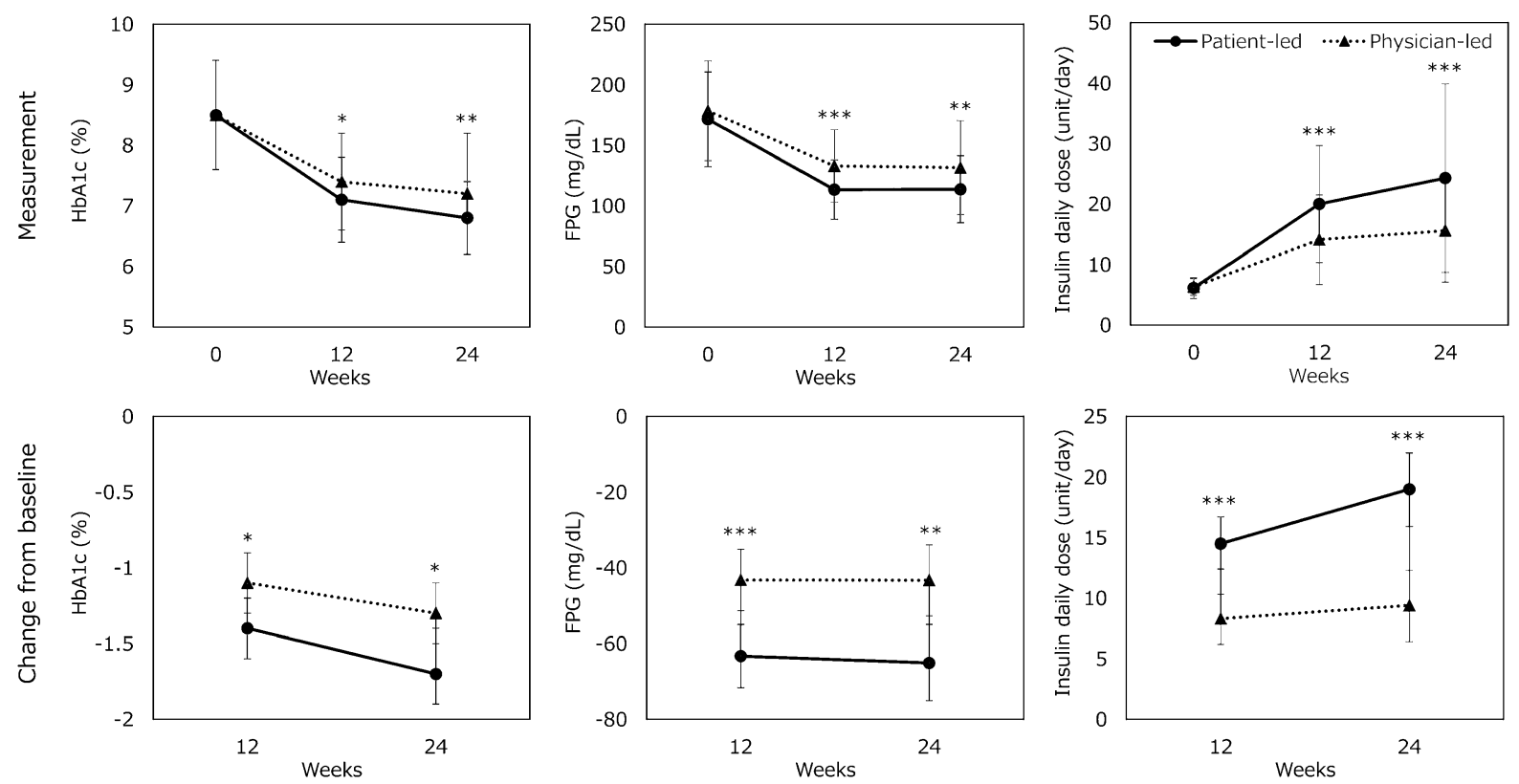

Fig. 3 Measurements and changes in clinical outcomes. Data are presented as the mean \pm standard deviation for measurements or the MMRM-adjusted mean $\pm 95 \%$ confidence interval for changes. Changes represent the change from baseline. $p$ values for between-group

(change from baseline was $0.9 \mathrm{~kg}(95 \%$ CI $0.3-1.5 \mathrm{~kg}$ ) and $0.9 \mathrm{~kg}$ (95\% CI $0.4-1.5 \mathrm{~kg}$ ) in the patient-led group and the physician-led group, respectively) and week 24 (change from baseline was $2.2 \mathrm{~kg}$ (95\% CI $1.4-3.0 \mathrm{~kg})$ and $1.6 \mathrm{~kg}(95 \% \mathrm{CI} 0.8-2.3 \mathrm{~kg})$ in the patient-led group and the physician-led group, respectively) (Table 2). However, no significant between-group difference was observed in the degree of body weight increase at week 12 (between-group difference was $0.0 \mathrm{~kg}(95 \% \mathrm{CI}$ -0.8 to $0.8 \mathrm{~kg}$ ), $p=0.998$ ) or week 24 (between-group difference was $0.6 \mathrm{~kg}(95 \% \mathrm{CI}$ -0.4 to $1.6 \mathrm{~kg}$ ), $p=0.232$ ).

\section{Safety Outcomes}

During the trial, 30 of the $59(50.8 \%)$ patients in the patient-led group and 35 of 61 (57.4\%) patients in the physician-led group reported adverse events (Table 3). The most common adverse event was hypoglycemia. Hypoglycemia was reported by 22 patients $(37.3 \%)$ in the comparisons were obtained using Student's $t$ test for measurements and MMRM for changes. ${ }^{*}$, $^{* *}$, and ${ }^{* * *}$ represent $p<0.05, p<0.01$, and $p<0.001$, respectively. $F P G$ fasting plasma glucose, $M M R M$ mixed-effects models for repeated-measures analysis

patient-led group and 23 patients (37.7\%) in the physician-led group, and no significant between-group difference was observed $(p=1.000)$. Severe hypoglycemia was not reported in either group throughout the trial. No significant between-group difference was observed in the frequency of other subcategories of hypoglycemia [documented symptomatic hypoglycemia in $11(18.6 \%)$ and 7 (11.5\%) patients (between-group $p=0.314$ ), asymptomatic hypoglycemia in 10 (16.9\%) and $8(13.1 \%)$ patients (between-group $p=0.615)$, probable symptomatic hypoglycemia in 11 $(18.6 \%)$ and $7(11.5 \%)$ patients (between-group $p=0.314)$, hypoglycemia-related symptoms in $5(8.5 \%)$ and $8(13.1 \%)$ patients (between-group $p=0.559)$, and nocturnal hypoglycemia in 0 $(0.0 \%)$ and $1(1.6 \%)$ patients (between-group $p=1.000)$ in the patient-led and the physicianled groups, respectively]. No significant between-group differences were observed in the frequencies of other adverse events. 
Table 2 Other clinical outcomes

\begin{tabular}{llll}
\hline Variable & Patient-led group & Physician-led group & $\boldsymbol{p}$ value \\
\hline Insulin self-injection adherence (\%) & & & \\
Week 0 to week 12 & $97.8 \pm 8.6$ & $97.4 \pm 9.0$ & 0.827 \\
Week 0 to week 24 & $97.4 \pm 8.5$ & $97.6 \pm 7.5$ & 0.888 \\
Week 12 to week 24 & $98.1 \pm 3.8$ & $98.3 \pm 4.8$ & 0.851 \\
Body weight (kg) & & & 0.518 \\
Baseline & $64.9 \pm 14.5(58)$ & $66.5 \pm 12.6(61)$ & 0.503 \\
Week 12 & $66.1 \pm 14.3(57)$ & $67.7 \pm 12.0(60)$ & \\
Change from baseline to week 12 & $0.9[0.3,1.5]$ & $0.9[0.4,1.5]$ & \\
Group difference in change & $0.0[-0.8,0.8]$ & & 0.998 \\
Week 24 & $67.5 \pm 14.5(55)$ & $68.5 \pm 12.4(58)$ & 0.697 \\
Change from baseline to week 24 & $2.2[1.4,3.0]$ & $1.6[0.8,2.3]$ & \\
Group difference in change & $0.6[-0.4,1.6]$ & & 0.232 \\
\hline
\end{tabular}

Data are presented as the mean \pm standard deviation (number of patients) for measurements or the mean [95\% confidence interval] for changes. $p$ values for between-group comparisons were obtained using Student's $t$ test for measurements and a generalized linear mixed-effects model for repeated measures for changes

$F P G$ fasting plasma glucose

\section{DISCUSSION}

The COMMIT-patient study was conducted to compare patient-led self-insulin titration with physician-led insulin titration in terms of their effects on patient QOL, treatment satisfaction, and self-efficacy in Japanese insulin-naïve T2DM patients. This is the first prospective, randomized controlled trial to estimate the effects of insulin initiation and titration on PROs (e.g., emotional distress, treatment satisfaction, and self-efficacy), safety, and clinical outcomes (e.g., glycemic control and body weight).

After initiating and titrating insulin, the PAID scores significantly improved from baseline to weeks 12 and 24 in both groups. The PAID is a questionnaire on diabetes-related emotional distress that measures the patient's psychological adaptation to diabetes. PAID scores have been reported to be negatively correlated to adherence to self-care behaviors such as diet therapy, exercise therapy, insulin use, SMBG, and glycemic control [8]. These self-care behaviors involving insulin injection included injecting the proper dose, injecting at the proper time, and titrating insulin based on SMBG. Greater adherence to these insulin selfmanagement behaviors correlates with a lower PAID score. By contrast, PAID scores tended to be higher in insulin-treated patients than in patients treated with other antidiabetic agents $[12,13]$, suggesting that insulin-treated DM patients have higher levels of emotional distress. Therefore, we hypothesized that PAID scores would increase after the initiation of insulin in insulin-naïve patients. However, in this study, PAID scores significantly decreased in both groups after insulin initiation. It may have been the case that insulin titration, regardless of whether the patient-led or the physician-led algorithm was followed, attenuated the patients' emotional distress, since patients in both groups were aware of the effects of the insulin via daily SMBG. HbA1c levels were clearly improved in both groups, no cases of severe hypoglycemia occurred, and only one case of nocturnal hypoglycemia was reported 
Table 3 Safety outcomes

\begin{tabular}{|c|c|c|c|}
\hline Adverse event & Patient-led group & Physician-led group & $p$ value \\
\hline Number of subjects in the safety analysis set & 59 & 61 & - \\
\hline Death & $0(0.0)$ & $0(0.0)$ & - \\
\hline Any adverse event & $30(50.8)$ & $35(57.4)$ & 0.583 \\
\hline Any serious adverse event & $1(1.7)$ & $1(1.6)$ & 1.000 \\
\hline Hypoglycemia & $22(37.3)$ & $23(37.7)$ & 1.000 \\
\hline Severe hypoglycemia & $0(0.0)$ & $0(0.0)$ & \\
\hline Documented symptomatic hypoglycemia & $11(18.6)$ & $7(11.5)$ & 0.314 \\
\hline Asymptomatic hypoglycemia & $10(16.9)$ & $8(13.1)$ & 0.615 \\
\hline Probable symptomatic hypoglycemia & $11(18.6)$ & $7(11.5)$ & 0.314 \\
\hline Hypoglycemia-related symptoms & $5(8.5)$ & $8(13.1)$ & 0.559 \\
\hline Nocturnal hypoglycemia & $0(0.0)$ & $1(1.6)$ & 1.000 \\
\hline Allergic rhinitis & $1(1.7)$ & $0(0.0)$ & 0.492 \\
\hline Insulin allergy & $0(0.0)$ & $1(1.6)$ & 1.000 \\
\hline Viral bronchitis & $0(0.0)$ & $1(1.6)$ & 1.000 \\
\hline Major depressive disorder & $0(0.0)$ & $1(1.6)$ & 1.000 \\
\hline Stress & $0(0.0)$ & $1(1.6)$ & 1.000 \\
\hline Hernia & $1(1.7)$ & $0(0.0)$ & 0.492 \\
\hline Dizziness & $1(1.7)$ & $0(0.0)$ & 0.492 \\
\hline Nausea & $0(0.0)$ & $1(1.6)$ & 1.000 \\
\hline Gastritis & $0(0.0)$ & $1(1.6)$ & 1.000 \\
\hline Gastroenteritis & $1(1.7)$ & $0(0.0)$ & 0.492 \\
\hline Macular edema & $1(1.7)$ & $0(0.0)$ & 0.492 \\
\hline Diarrhea & $2(3.4)$ & $1(1.6)$ & 0.616 \\
\hline Hepatic dysfunction & $0(0.0)$ & $1(1.6)$ & 1.000 \\
\hline Hunger & $1(1.7)$ & $0(0.0)$ & 0.492 \\
\hline Neck pain & $1(1.7)$ & $0(0.0)$ & 0.492 \\
\hline Bone fracture & $1(1.7)$ & $0(0.0)$ & 0.492 \\
\hline Autoimmune pancreatitis & $0(0.0)$ & $1(1.6)$ & 1.000 \\
\hline Lowering of systolic blood pressure & $0(0.0)$ & $1(1.6)$ & 1.000 \\
\hline Upper airway inflammation & $0(0.0)$ & $1(1.6)$ & 1.000 \\
\hline Upper airway cough syndrome & $1(1.7)$ & $0(0.0)$ & 0.492 \\
\hline
\end{tabular}


Table 3 continued

\begin{tabular}{llll}
\hline Adverse event & Patient-led group & Physician-led group & $\boldsymbol{p}$ value \\
\hline Lowering of ventricular wall motion & $0(0.0)$ & $1(1.6)$ & 1.000 \\
Wound infection & $1(1.7)$ & $0(0.0)$ & 0.492 \\
Herpes zoster & $1(1.7)$ & $0(0.0)$ & 0.492 \\
Enteritis & $0(0.0)$ & $1(1.6)$ & 1.000 \\
Headache & $0(0.0)$ & $1(1.6)$ & 1.000 \\
Urinary tract infection & $1(1.7)$ & $0(0.0)$ & 0.492 \\
Dermatitis & $0(0.0)$ & $1(1.6)$ & 1.000 \\
Edema & $1(1.7)$ & $0(0.0)$ & 0.492 \\
Abdominal distention & $0(0.0)$ & $1(1.6)$ & 1.000 \\
Drug eruption & $0(0.0)$ & $1(1.6)$ & 1.000 \\
Cystitis & $1(1.7)$ & $1(1.6)$ & 1.000 \\
Bone fracture (serious) & $1(1.7)$ & $0(0.0)$ & 0.492 \\
Autoimmune pancreatitis (serious) & $0(0.0)$ & $1(1.6)$ & 1.000 \\
\hline
\end{tabular}

Data are presented as number of patients (\%). $p$ values for between-group comparisons were obtained using Fisher's exact test

during the study. Indeed, the change in PAID score from baseline to week 24 was positively correlated with the change in HbA1c from baseline to week $24(r=0.19)$. We had also hypothesized that PAID scores would be lower in the patient-led group than in the physicianled group, since participants in the patient-led group adjusted the insulin dose themselves based on SMBG, distinct treatment goals, and their understanding of the relationship between dietary intake and required insulin dose. Although the improvement in PAID scores was greater in the patient-led group, no significant between-group difference was observed in this study. Two recent studies reported similar results. The Take Control study, which compared patient-led with physician-led insulin titration in European T2DM patients, reported that emotional distress (as measured via the Diabetes Distress Scale) improved in both groups, and that there was no significant between-group difference [5]. The ITAS study conducted in Italy also demonstrated that PAID scores were reduced in both patient-managed and physician-managed groups, and found no significant between-group difference [14]. Together, these results suggest that insulin initiation by patient-led self-titration could achieve comparable improvements in emotional distress to physician-led insulin titration, especially in European countries and Japan.

Treatment satisfaction, as measured by the DTSQ, improved significantly from baseline to weeks 12 and 24 in both groups. While treatment satisfaction was higher in the patient-led group, no significant between-group difference was observed. Similarly, the ATLAS study [2] and ITAS study [14] showed that insulin initiation improved treatment satisfaction, as measured by DTSQ, and reported no differences between physician-led and patient-led titration, thus demonstrating that patient-led insulin selftitration did not compromise treatment satisfaction [2]. Together, these results suggest that patient-led insulin titration could yield similar treatment satisfaction to physician-led insulin titration if conducted safely and effectively. 
Confidence in treatment is an important factor in patient self-management. The Take Control study [5] and the ITAS study [14] compared patient empowerment, as measured by the Diabetes Empowerment Scale, between patient-led and physician-led titration groups. Empowerment scores increased from baseline to week 24 in both groups in both studies, meaning that patients' confidence in their diabetes management increased with patient-led insulin titration. The ITSS is a questionnaire that assesses self-efficacy in insulin treatment [11]. Domain 2 scores relate to the patient's confidence with insulin titration, and domain 3 scores concern the patient's confidence with glycemic control. Both domains were significantly higher in the patient-led group at weeks 12 and 24. This may be because patients in the patient-led group obtained more detailed instruction on insulin titration and achieved confidence in their insulin titration based on SMBG. This finding highlights the importance of insulin self-titration at the start of insulin therapy in T2DM patients. Conversely, domain 1 scores (regarding the patient's confidence in the insulin injection procedure) and domain 4 scores (regarding the patient's confidence in their ability to cope with hypoglycemia) did not show any significant between-group differences. Because knowledge of the insulin injection procedure and how to manage hypoglycemia was required in both the patientled and the physician-led groups, these results may demonstrate that the subjects who participated in this study were well educated and confident in their abilities, regardless of their treatment allocation. The ITSS total score at week 24 was positively correlated with SMBG adherence $(r=0.24)$ and negatively correlated with HbA1c $(r=-0.27)$. SMBG adherence was positively correlated with insulin injection adherence $(r=0.36)$ and was negatively correlated with HbA1c $(r=-0.25)$. These results suggest that increased self-efficacy could improve SMBG and insulin injection adherence and glycemic control, and that the ITSS could be a useful measurement tool to assess the success or failure of patient-led insulin titration.

In this study, greater reductions in HbA1c and FPG were observed in the patient-led group compared with the physician-led group. The ATLAS study also reported greater decreases in HbA1c and FPG in the patient-led group compared with the physician-led group [2], while the Take Control study showed a larger decrease in HbA1c but not in FPG in the patient-led group compared with the physician-led group [5], and the ITAS study reported that the patient-led group had noninferior but not superior values of HbA1c and FPG compared with the physician-led group [14].

In this study, the insulin titration algorithm was mainly based on the algorithm used in the ATLAS study $[2,15]$, in which a FPG of less than $6.0 \mathrm{mmol} / \mathrm{L}$ was targeted. In addition to the insulin titration algorithm used in the ATLAS study, this study classified FPG values of 6.0-$9.0 \mathrm{mmol} / \mathrm{L}$ into four steps $(6,7,8$, and $9 \mathrm{mmol} /$ L) for insulin dose titration to avoid inducing hypoglycemia through excess insulin administration. Patients in the physician-led group were asked to titrate the insulin dose at every observation point, while patients in the patient-led group titrated the insulin dose more frequently themselves based on SMBG. As a result, while both groups started at approximately 6 units/day of insulin, the change in the insulin daily dose from baseline to week 24 was twice as large in the patient-led group (19.0 units/day) than in the physician-led group (9.4 units/day). Regardless of the differences in the insulin titration algorithms and the changes in the insulin daily dose, insulin injection adherence was as high as $>97 \%$ throughout the study in both groups, and no significant between-group difference was observed. These results suggest that insulin self-titration can be managed without compromising insulin injection adherence.

Despite the greater increase in insulin daily dose in the patient-led group, body weight increased similarly in both groups in this study. The ATLAS study also found that body weight increased similarly in both groups despite a greater increase in insulin daily dose in the patient-led group [2]. The Take Control and ITAS studies showed similar increases in body weight along with similar increases in insulin daily dose $[5,14]$. 
Greater increases in insulin daily dose and body weight and greater improvements in HbA1c and FPG were observed in the patientled group compared with the physician-led group in this study and the ATLAS study, while similar increases in insulin daily dose and body weight and similar improvements in glycemic control were reported for the patient-led and physician-led groups in the Take Control and ITAS studies. Since this study and the ATLAS study were conducted in Asian countries, including Japan, while the Take Control study and the ITAS study were conducted in European countries, ethnicity may be a reason for the interstudy differences in the effects of patientled and physician-led insulin titration on clinical outcomes. Further international clinical trials are required to compare patient-led and physician-led insulin titration across countries, regions, and ethnicities.

Despite the greater increase in insulin daily dose and greater reductions in FPG and HbA1c in the patient-led insulin titration group, the frequency of hypoglycemia was almost the same in both groups. Severe hypoglycemia was not reported in either group during the study, and there was no significant between-group difference in the frequencies of other subcategories of hypoglycemia (documented symptomatic hypoglycemia, asymptomatic hypoglycemia, probable symptomatic hypoglycemia, hypoglycemia-related symptoms, and nocturnal hypoglycemia).

Taken together, for patients in both the patient-led insulin titration group and the physician-led insulin titration groups, the initiation of insulin therapy in insulin-naïve Japanese T2DM patients ultimately resulted in effective glycemic control without severe hypoglycemia. The patient-led insulin titration group was superior in terms of glycemic control and self-efficacy. Even though healthcare providers intend to provide the best possible treatment, patients can be reluctant to initiate and escalate therapy. The gap between clinical best practice and patient perception is termed "clinical inertia" [16]. Clinical inertia often occurs during insulin initiation. Even when insulin treatment is initiated, the injection of smaller doses than recommended is frequently observed. This results in poor glycemic control. One reason for this clinical inertia regarding insulin therapy might be physician-led insulin titration. SMBG and insulin self-titration are well established in T1DM treatment, as insulin injection is necessary in T1DM. The results of the ATLAS [2] and Take Control [5] studies and the present study clearly demonstrate that SMBG and insulin self-titration are also effective for T2DM patients and can be conducted safely. Insulin self-titration based on SMBG promotes patient involvement and confidence in their DM therapy and empowers them. Self-management may help combat clinical inertia regarding insulin treatment for T2DM patients.

This study had several limitations. First, it did not reach the target sample size. In this study, although we planned to enroll 250 patients, 125 patients were ultimately enrolled. There are several possible reasons for this undersized sample. First, among injectable antidiabetic agents, the use of glucagon-like peptide-1 receptor agonists (GLP-1 RAs) has gradually increased whereas the use of insulin has decreased year-on-year in Japan [17], probably because GLP-1 RAs have several advantages over insulin, such as weight-loss efficacy, cardiovascular benefits, and a low risk of hypoglycemia $[18,19]$. The number of patients with T2DM who wanted to initiate insulin therapy may therefore have been unexpectedly low. Another possible reason is that there is often a psychological barrier to insulin initiation in patients with T2DM [20]. The additional intervention associated with participation in this study may have led to an even greater psychological barrier, resulting in a failure to obtain informed consent within the limited recruitment period. Also, the recruitment period was limited due to competitive enrollment with other studies during the study period. A posthoc power calculation was performed using data on treatment effect and the SD observed in this study for the primary endpoint. As a result, a much larger sample size would have been required for the primary outcome measure $(n=133,758)$ to detect a treatment effect (group difference mean $=-0.13$ and standard deviation =12). Even with a longer recruitment period, it would therefore have been difficult to 
meet the target sample size in this study, and it would be difficult to achieve in any planned new trial. However, this study clearly demonstrated favorable effects of patient-led self-titration on self-efficacy and glycemic control. Finally, this was an open-label trial, and all the patients were Japanese. Although the main aspects of insulin self-titration are similar regardless of the Asian country considered, the results of this study cannot be generalized to Asian T2DM patients. Further country-specific investigations and an international trial are required.

\section{CONCLUSIONS}

Self-measurement of blood glucose and self-titration of insulin enhanced patient self-efficacy without compromising their emotional distress or treatment satisfaction. Also, insulin self-titration was safe and effective, resulting in better glycemic control with no events of severe hypoglycemia. Further, while patient-led and physician-led titration resulted in similar levels of emotional distress and treatment satisfaction, self-titration enhanced patient self-efficacy.

\section{ACKNOWLEDGEMENTS}

The authors thank all of the clinical staff for their assistance in the execution of the study, and all the participants of this study.

Funding. This study was financially supported by Sanofi K.K. The journal's Rapid Service Fee and the fee charged by Soiken Inc. for technical assistance with launching and executing the study and for facilitating the preparation of the manuscript were also covered by Sanofi K.K.

Medical Writing and/or Editorial Assistance. The authors thank Soiken Inc. for their technical assistance with the launch and execution of the study and their support with the medical writing of the manuscript (the fee from Soiken Inc. was covered by Sanofi K.K.).
Authorship. All named authors meet the International Committee of Medical Journal Editors (ICMJE) criteria for authorship for this article, take responsibility for the integrity of the work as a whole, and have given their approval for this version to be published.

Author Contributions. HI contributed to the conception and design of the study, subject enrollment, data acquisition, and manuscript drafting and revision. HN contributed to the design of the study, subject enrollment, and data acquisition. NK, DU, DS, YO, and DS contributed to subject enrollment and data acquisition. YS contributed to the design of the study and the statistical analysis.

Disclosures. Hitoshi Ishii has received honoraria from Ono Pharmaceutical Co., Mitsubishi Tanabe Pharma, MSD, Novo Nordisk Pharma Ltd., Daiichi Sankyo, Takeda Pharmaceutical Co., Eli Lilly Japan, Sanofi, and Sumitomo Dainippon Pharma. Nozomu Kamei has received lecture fees from Sanofi K.K. and Sumitomo Dainippon Pharma Co., Ltd., and a grant from Astellas Pharma Inc. Daigaku Uchida has received lecture fees from Sanofi K.K., Novo Nordisk Pharma Ltd., Eli Lilly and Company, Mitsubishi Tanabe Pharma Co., MSD K.K., and Takeda Pharmaceutical Co., Ltd. Hiroki Nakajima, Daisuke Suzuki, Yasuhiro Ono, Yasunori Sato, and Dai Shimoto declare no conflict of interest.

Compliance with Ethics Guidelines. This study and its protocols were approved by the institutional review board of each participating institution according to the Ethical Guidelines for Medical and Health Research Involving Human Subjects issued by the Ministry of Health, Labour and Welfare in Japan. The names of the ethics committees that approved the study are shown in Table S1 of the "Supplementary Information." This study was first registered in the University Hospital Medical Information Network Clinical Trial Registry (UMIN-CTR) (registration number: UMIN000020316). The study was conducted in accordance with the Declaration of Helsinki, Ethical Guidelines for Medical and Health 
Research Involving Human Subjects, and other current legal regulations in Japan. Written informed consent was obtained prior to treatment from all enrolled patients who met the eligibility criteria.

Data Availability. The datasets generated and/or analyzed during the current study are not publicly available due to the lack of a statement in the study protocol and informed consent documents that would enable data sharing with a third party after the end of the study, as well as a lack of approval for data sharing by the institutional review board of each participating institution.

Open Access. This article is licensed under a Creative Commons Attribution-NonCommercial 4.0 International License, which permits any non-commercial use, sharing, adaptation, distribution and reproduction in any medium or format, as long as you give appropriate credit to the original author(s) and the source, provide a link to the Creative Commons licence, and indicate if changes were made. The images or other third party material in this article are included in the article's Creative Commons licence, unless indicated otherwise in a credit line to the material. If material is not included in the article's Creative Commons licence and your intended use is not permitted by statutory regulation or exceeds the permitted use, you will need to obtain permission directly from the copyright holder. To view a copy of this licence, visit http://creativecommons.org/licenses/by$\mathrm{nc} / 4.0 /$.

\section{REFERENCES}

1. Davies M, Storms F, Shutler S, et al. Improvement of glycemic control in subjects with poorly controlled type 2 diabetes: comparison of two treatment algorithms using insulin glargine. Diabetes Care. 2005;28:1282-8.

2. Garg SK, Admane K, Freemantle N, et al. Patient-led versus physician-led titration of insulin glargine in patients with uncontrolled type 2 diabetes: a randomized multinational ATLAS study. Endocr Pract. 2015;21:143-57.
3. Khunti K, Davies MJ, Kalra S. Self-titration of insulin in the management of people with type 2 diabetes: a practical solution to improve management in primary care. Diabetes Obes Metab. 2013;15: $690-700$.

4. Chraibi A, Al-Herz S, Nguyen BD, et al. An RCT investigating patient-driven versus physician-driven titration of BIAsp 30 in patients with type 2 diabetes uncontrolled using NPH insulin. Diabetes Ther. 2017;8:767-80.

5. Russell-Jones D, Dauchy A, Delgado E, et al. Take Control: a randomized trial evaluating the efficacy and safety of self- versus physician-managed titration of insulin glargine $300 \mathrm{U} / \mathrm{mL}$ in patients with uncontrolled type 2 diabetes. Diabetes Obes Metab. 2019;21:1615-24.

6. Bradley CL. The diabetes treatment satisfaction questionnaire: DTSQ. Handb Psychol Diabetes. 1994;111:132.

7. EuroQol Group. EuroQol-a new facility for the measurement of health-related quality of life. Health Policy. 1990;16:199-208.

8. Polonsky WH, Anderson BJ, Lohrer PA, et al. Assessment of diabetes-related distress. Diabetes Care. 1995;18:754-60.

9. Ishii $H$, Welch $G$, Jacobson A, et al. The Japanese version of Problem Area in Diabetes Scale: a clinical and research tool for the assessment of emotional functioning among diabetes patients. Diabetes. 1999;48:A319.

10. Ishii H, Bradley C, Riazi A, et al. The Japanese version of the Diabetes Treatment Satisfaction Questionnaire (DTSQ): translation and clinical evaluation. J Clin Exp Med. 2000;192:809.

11. Nakaue J, Koizumi M, Nakajima H, et al. Development of a self-efficacy questionnaire, "Insulin Therapy Self-efficacy Scale (ITSS)", for insulin users in Japanese: The Self-Efficacy-Q study. J Diabetes Investig. 2019;10:358-66.

12. Hayashino $Y$, Okamura S, Matsunaga S, et al. The association between problem areas in diabetes scale scores and glycemic control is modified by types of diabetes therapy: Diabetes Distress and Care Registry in Tenri (DDCRT 2). Diabetes Res Clin Pract. 2012;97:405-10.

13. Hayashino Y, Okamura S, Tsujii S, et al. Association between diabetes distress and all-cause mortality in Japanese individuals with type 2 diabetes: a prospective cohort study (Diabetes Distress and Care Registry in Tenri [DDCRT 18]). Diabetologia. 2018;61:1978-84. 
14. Bonadonna RC, Giaccari A, Buzzetti R, et al. Comparable efficacy with similarly low risk of hypoglycaemia in patient- vs physician-managed basal insulin initiation and titration in insulin-naive type 2 diabetic subjects: the Italian Titration Approach Study. Diabetes Metab Res Rev. 2020;36:e3304.

15. ATLAS Study Group. Titration of insulin glargine in patients with type 2 diabetes mellitus in Asia: physician- versus patient-led? Rationale of the Asian Treat to Target Lantus Study (ATLAS). Diabetes Technol Ther. 2011;13:67-72.

16. Khunti K, Millar-Jones D. Clinical inertia to insulin initiation and intensification in the UK: a focused literature review. Prim Care Diabetes. 2017;11:3-12.

17. Japan Diabetes Clinical Data Management Study Group. Basic summary data 2019. Available at http://jddm.jp/data/index-2019/. Accessed 27 Oct 2020 .

18. Cernea S, Raz I. Therapy in the early stage: incretins. Diabetes Care. 2011;34(Suppl. 2):S264-271.

19. Kaku K, Yamada Y, Watada H, et al. Safety and efficacy of once-weekly semaglutide vs additional oral antidiabetic drugs in Japanese people with inadequately controlled type 2 diabetes: a randomized trial. Diabetes Obes Metab. 2018;20: 1202-12.

20. Hosomura N, Malmasi S, Timerman D, et al. Decline of insulin therapy and delays in insulin initiation in people with uncontrolled diabetes mellitus. Diabet Med. 2017;34:1599-602. 